\title{
An insidious case of Aspergillus Niger graft aortitis after cardiac surgery
}

\author{
Matteo Marro ${ }^{1}$, Francesco Atzeni ${ }^{1}$, Michele La Torre ${ }^{1}$, Matteo Attisani ${ }^{1}$, Stefania Belloro ${ }^{1}$, \\ Francesco De Rosa ${ }^{1}$, and Mauro Rinaldi ${ }^{1}$ \\ ${ }^{1}$ Azienda Ospedaliero Universitaria Città della Salute e della Scienza di Torino
}

April 27, 2020

\begin{abstract}
Fungal endocarditis/aortitis is an uncommon yet emerging entity accounting for $2 \%$ to $4 \%$ of all cases of infective endocarditis and continues to be associated with a poor prognosis. We present here the first case of polyethylene-terephthalate (PETE) graft aortitis caused by A. Niger, a rare fungal agent responsible for the Aspergillus aortitis. Early diagnosis with frequent transoesophageal echocardiography (TEE) and a prompt surgical intervention coupled with optimal antifungal therapy are still the only option to reduce the exceedingly high mortality and morbidity.
\end{abstract}

\begin{abstract}
:
Fungal endocarditis/aortitis is an uncommon yet emerging entity accounting for $2 \%$ to $4 \%$ of all cases of infective endocarditis and continues to be associated with a poor prognosis. We present here the first case of polyethylene-terephthalate (PETE) graft aortitis caused by A. Niger, a rare fungal agent responsible for the Aspergillus aortitis. Early diagnosis with frequent transoesophageal echocardiography (TEE) and a prompt surgical intervention coupled with optimal antifungal therapy are still the only option to reduce the exceedingly high mortality and morbidity.
\end{abstract}

\section{INTRODUCTION}

Aspergillus is an opportunistic nosocomial fungus generally associated with a high mortality rate ${ }^{1}$. While $A$. fumigatus has been reported to be the most frequent cause of aspergillosis, A. Niger has been rarely associated with infection and only few cases have been reported in patients who have recently undergone valvular heart surgery ${ }^{2-5}$ and pacemaker implantation ${ }^{6}$, as well as in patients with neoplastic diseases, intravenous treatment or drug addiction, long-term parenteral feeding and immunosuppression.

\section{CASE REPORT}

A 38-year-old man with a history of congenital aortic valve stenosis underwent a mechanical aortic valve replacement (21 mm SJ Regent) with aortotomy's closure by a pericardium patch at an external center. The postoperative period was impacted by sporadic serotine fever treated with empiric antibiotic therapy but two months after surgery he was referred to our center due to enduring low-grade fever and mild leucocytosis; apart from these signs his physical examination was unremarkable and there were no signs of petechiae, splenomegaly or clubbing. All microbiological evaluations were uneventful, transoesophageal echocardiography (TEE) examination showed no endocarditis signs but computerized tomography (CT) scan unveiled a pseudo-aneurysmatic degeneration ( $6.5 \times 5.3$ centimetres) on the aortic wall in correspondence of the aortotomy's suture. Reoperation was decided upon and he underwent an ascending aortic replacement with polyethylene-terephthalate (PETE) $28 \mathrm{~mm}$ Hemashield graft; no suggestive signs of active infection were detected in the pericardial patch, therefore coltural examination of the sample was not performed. He 
was discharged in excellent condition but after 7 months he was re-admitted to our center for new onset of fever and malaise, distal leg and spleen embolization and high levels of inflammatory biomarkers. In cardiac auscultation, cardiac sounds were rhythmic and metallic valve sound was heard. The TEE showed a regular aortic prosthesis, the CT scan a peri-aortic exudation without enhancement and the positron emission tomography (PET) confirmed an aspecific postoperative inflammatory exudate around the graft. All four blood culture sets were negative as were serology for rickettsia and Q fever; the patient was treated empirically with Vancomycin and Ceftriaxone and thus fever subsided and two different TEEs confirmed no sign of endocarditis. Although three weeks of antibiotic therapy, inflammatory biomarkers increased again and for the first time TEE revealed two mobile masses $(15 \times 16 \mathrm{~mm}$ each), suggestive of vegetations, with a stalk at the ascending aorta graft wall (Figure 1A, B; Supp Video) one centimetre around over the right coronary ostium without evidence of valvular damage; a new CT scan confirmed the diagnosis showing a $8 \mathrm{~mm}$ filling defect in the same position (Figure 1C, D). All six blood culture sets were negative and with the diagnosis of culture-negative graft aortitis caspofungin was administered due to the $\beta$-d-glucan (BDG) positivity; thus a new surgery was performed with aortic homograft root implantation: intraoperative exploration confirmed the aortitis of the PETE prothesis and the resected material was sent to our microbiology laboratories that revealed A. Niger infection. The patient's hemodynamic has been supported by dobutamine for 8 days and heart rate by temporary epicardial ventricular pacing due to third-degree atrioventricular block with following spontaneous recovery with sinus rhythm. The patient received parenteral liposomal amphotericin-B $(5 \mathrm{mg} / \mathrm{kg}$ per day) for 45 days followed by oral voriconazole for another 6 months with good clinical evolution. The six-month follow-up TEE showed no endocarditis/aortitis and all the inflammatory biomarkers were negative.

\section{COMMENT}

Fungal endocarditis/aortitis is an uncommon yet emerging entity accounting for $2 \%$ to $4 \%$ of all cases of infective endocarditis and continues to be associated with a poor prognosis ${ }^{7}$.A. Fumigatus is the most common fungal agent responsible for the Aspergillus aortitis and its diagnosis is very difficult. Blood cultures are negative in over $50 \%$ of cases, $\beta$-D-glucan has a sensitivity and specificity of $69.9 \%$ and $87.1 \%$ respectively ${ }^{7}$ and the infection may be overlooked by transthoracic echocardiography as it localizes in the ascending aorta. This represents the fourth case of $A$. Niger aortitis ${ }^{4,5,8}$, the first one involving a PETE graft. The only predisposing factor for A. Nigerinfection in this patient was the first open heart surgery. AlthoughA. Niger mycotic endocarditis/aortitis is rare among immunocompetent patients, the diagnosis should be considered for all patients with fever and an unidentified infection origin who have had heart surgery. The case emphasizes the role of serial TEEs in the diagnosis and management of these patients particularly with negative cultures and it illustrates the possible insidious course of fungal endocarditis/aortitis that continues to be associated with a poor prognosis. Early diagnosis and a prompt surgical intervention coupled with optimal antifungal therapy are still our only option to reduce the exceedingly high mortality and morbidity.

\section{Acknowledgments and Disclosures:none}

\section{References:}

1. Glampedakis E, Cassaing S, Fekkar A, et al. Invasive aspergillosis due to Aspergillus section Usti: a multicenter retrospective study. Clin Infect Dis. 2020.

2. Moore RS, Hasleton PS, Lawson RA, Stanbridge TN. Aspergillus niger endocarditis complicating aortic tissue valve replacement. Thorax. 1984;39(1):76-77.

3. Kreiss Y, Vered Z, Keller N, Kochva I, Sidi Y, Gur H. Aspergillus niger endocarditis in an immunocompetent patient: an unusual course.Postgrad Med J. 2000;76(892):105-106.

4. Duygu H, Nalbantgil S, Ozerkan F, Kirilmaz B, Yagdi T. Aspergillus niger aortitis after aortic valve replacement diagnosed by transesophageal echocardiography. Echocardiography.2006;23(5):405-406.

5. Jamieson RW, Wallace WA, Din JN, Raza Z. Acute aortic occlusion with sudden paraplegia secondary to Aspergillus niger embolism from Aspergillus niger aortitis. J Vasc Surg. 2011;54(5):1472-1474. 
6. Ho IC, Milan DJ, Mansour MC, et al. Fungal infection of implantable cardioverter-defibrillators: case series of five patients managed over 22 years. Heart Rhythm. 2006;3(8):919-923.

7. Ammannaya GKK, Sripad N. Fungal endocarditis: what do we know in 2019? Kardiol Pol. 2019;77(78):670-673.

8. Noordally SO, Sohawon S, De Bels D, Duttmann R, Gottignies P, Devriendt J. Late onset of Aspergillus aortitis presenting as femoral artery embolism following coronary artery bypass graft surgery.Acta Medica (Hradec Kralove). 2011;54(4):175-176.

\section{Figure legends:}

Figure 1. TEE showing the mobile masses (in the yellow circle) suggestive of vegetations with a stalk at the ascending aorta graft wall (A : $130^{\circ}$, mid-esophageal, long-axis view; $\mathbf{B}: \mathrm{X}$-plane view); CT scan revealing a filling defect (in the yellow circle) in the aortic graft ( $\mathbf{C}$ : axial plane; $\mathbf{D}$ : coronal plane). LA, left atrium; $\mathrm{LV}$, left ventricle.

Supp Video. TEE shows the vegetations of the ascending aorta graft (in the left mid-esophageal, long-axis view; in the right $\mathrm{X}$-plane view)

Hosted file Fig 1 .pptxavailableathttps : //authorea.com/users/315254/articles/445609 - an - insidious case - of - aspergillus - niger - graft - aortitis - after - cardiac - surgery 